\title{
Front Matter: Volume 11233
}

, "Front Matter: Volume 11233," Proc. SPIE 11233, Optical Fibers and Sensors for Medical Diagnostics and Treatment Applications XX, 1123301 (6 March 2020); doi: 10.1117/12.2569725

SPIE. Event: SPIE BiOS, 2020, San Francisco, California, United States 


\section{PROGRESS IN BIOMEDICAL OPTICS AND IMAGING}

\section{Optical Fibers and Sensors for Medical Diagnostics and Treatment Applications XX}

Israel Gannot

Editor

1-2 February 2020

San Francisco, California, United States

Sponsored and Published by

SPIE

Volume 11233 
The papers in this volume were part of the technical conference cited on the cover and title page. Papers were selected and subject to review by the editors and conference program committee. Some conference presentations may not be available for publication. Additional papers and presentation recordings may be available online in the SPIE Digital Library at SPIEDigitalLibrary.org.

The papers reflect the work and thoughts of the authors and are published herein as submitted. The publisher is not responsible for the validity of the information or for any outcomes resulting from reliance thereon.

Please use the following format to cite material from these proceedings:

Author(s), "Title of Paper," in Optical Fibers and Sensors for Medical Diagnostics and Treatment Applications XX, edited by Israel Gannot, Proceedings of SPIE Vol. 11233 (SPIE, Bellingham, WA, 2020) Seven-digit Article CID Number.

ISSN: 1605-7422

ISSN: 2410-9045 (electronic)

ISBN: 9781510632295

ISBN: 9781510632301 (electronic)

Published by

SPIE

P.O. Box 10, Bellingham, Washington 98227-0010 USA

Telephone +1 3606763290 (Pacific Time) · Fax +1 3606471445

SPIE.org

Copyright (c) 2020, Society of Photo-Optical Instrumentation Engineers.

Copying of material in this book for internal or personal use, or for the internal or personal use of specific clients, beyond the fair use provisions granted by the U.S. Copyright Law is authorized by SPIE subject to payment of copying fees. The Transactional Reporting Service base fee for this volume is $\$ 21.00$ per article (or portion thereof), which should be paid directly to the Copyright Clearance Center (CCC), 222 Rosewood Drive, Danvers, MA 01923. Payment may also be made electronically through CCC Online at copyright.com. Other copying for republication, resale, advertising or promotion, or any form of systematic or multiple reproduction of any material in this book is prohibited except with permission in writing from the publisher. The CCC fee code is $1605-$ $7422 / 20 / \$ 21.00$.

Printed in the United States of America by Curran Associates, Inc., under license from SPIE.

Publication of record for individual papers is online in the SPIE Digital Library.

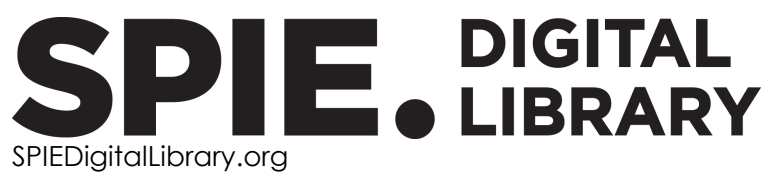

Paper Numbering: Proceedings of SPIE follow an e-First publication model. A unique citation identifier (CID) number is assigned to each article at the time of publication. Utilization of CIDs allows articles to be fully citable as soon as they are published online, and connects the same identifier to all online and print versions of the publication. SPIE uses a seven-digit CID article numbering system structured as follows:

- The first five digits correspond to the SPIE volume number.

- The last two digits indicate publication order within the volume using a Base 36 numbering system employing both numerals and letters. These two-number sets start with $00,01,02,03,04$, 05, 06, 07, 08, 09, 0A, OB ... 0Z, followed by 10-1Z, 20-2Z, etc. The CID Number appears on each page of the manuscript. 


\section{Contents}

$\begin{array}{ll}\text { vii } & \text { Authors } \\ \text { ix } & \text { Conference Committee }\end{array}$

FIBER OPTIC SENSORS: DEVELOPMENT AND CHARACTERIZATION I

$1123302 \quad$ Evanescent wave sensor using cellulose nanocrystals composite fiber coating for humidity measurement [11233-1]

$1123305 \quad$ Non-contact endoscopic temperature measurement [11233-4]

1123306 Sensitivity analysis of $\mathrm{TiO}_{2}$ coated fibre Bragg grating sensor for far infrared detection of chemicals [11233-5]

FIBER OPTIC TOOLS FOR MEDICAL APPLICATIONS I

1123309 A biomimetic optical approach to skin cancer detection [11233-9]

ENVIRONMENTAL SENSING

$11233 \mathrm{OH} \quad$ Water Soluble Fraction (WSF) contaminant detection using machine-learning and Absorbance-Transmission Excitation Emission Matrix (A-TEEM) spectroscopy [1 1233-55]

FIBER OPTIC TOOLS FOR MEDICAL APPLICATIONS II

11233 OK Characterizing collapse during obstructive sleep apnea through fiber optic manometry [11233-19]

$11233 \mathrm{OL} \quad$ Photosensitizing effect of Photolon using optical fiber probe to laser-irradiate thyroid cancer cells: oxidative stress-directed cell death [1 1233-20]

1123300 Stability diagnosis of orthopedic implants based on resonance frequency analysis with fiber transmission of nanosecond laser pulse and acceleration sensor [11233-23]

FIBER OPTIC SENSORS: DEVELOPMENT AND CHARACTERIZATION II

$112330 Q \quad$ PDMS-based Bragg diffraction grating glucose sensor integrable in a contact lens [1 1233-25] 
11233 OS Fiber bundles with integrated bandpass and notch filters for in-vivo Raman spectroscopy [1 1233-27]

11233 OT 2D temperature sensing obtained by multiplexing of optical backscattering reflectometry [1 1233-28]

11233 OU Spatial multiplexing of refractive index distributed sensors by means of high-scattering MgO nanoparticle doped optical fiber [11233-29]

FIBER OPTIC TOOLS FOR MEDICAL APPLICATIONS III

11233 OY Fiber optic biosensor based on polydimethylsiloxane (PDMS) and bioactive lipids [11233-33]

FIBER OPTIC TOOLS FOR MEDICAL APPLICATIONS IV

$11233 \mathrm{OZ} \quad$ Tapered fiber sensor usable for head and neck cancer screening [1 1233-34]

$1123311 \quad$ Mid-infrared spectroscopy with a fiber-coupled tuneable quantum cascade laser for glucose sensing [11233-36]

1123313 Single fiber OCT imager for breast tissue classification based on deep learning [1 1233-38]

FIBER OPTIC SENSORS: DEVELOPMENT AND CHARACTERIZATION III

1123315 Transmission properties of dielectric-coated hollow optical fibers based on Ni-Ti tube [1 1233-40]

1123316 Twisting compensation of optical multicore fiber shape sensors for flexible medical instruments [11233-41]

1123317 Photonic crystal fiber refractive index sensor with ultra-wide detection range based on surface plasmon resonance [11233-42]

1123318 3D shape sensing of an epidural needle based on simultaneous spatial multiplexing of optical backscattering reflectometry [11233-43]

1123319 Sensitivity analysis of a square shape apodize fibre Bragg grating chemical sensor assisted by high refractive index bi-directional coupler on both sides [11233-44]

$112331 \mathrm{~A} \quad$ Study and analysis of nature of cladding modes in tilted fiber Bragg gratings and their correlation with grating parameters for design of optimized sensors [1 1233-45] 


\section{POSTER SESSION}

11233 1B Performance comparison of the fiber axicon probe having extended Bessel focus with cleaved fiber tip probe [11233-46]

$11233 \mathrm{IE} \quad$ Rapid detection of Escherichia coli in aqueous samples by metabolic induced change in pH [11233-49]

$11233 \mathrm{IF} \quad$ Reflection-mode fiber-optic temperature sensing probe based on quantum dots filled micro-cavity [11233-50]

$112331 \mathrm{H} \quad$ Measurements of coupling efficiency of high power Er:YAG laser in different types of optical fibers [11233-52]

$112331 \mathrm{~J} \quad$ Silver mirror coated deep seated negative axicon optical fiber tip for highly efficient Bessel-Gauss beam generation [11233-54]

$112331 \mathrm{~K} \quad$ Fast response time fiber optical pH and oxygen sensors [1 1233-56] 
Proc. of SPIE Vol. 11233 1123301-6

\section{Downloaded From: https://www.spiedigitallibrary.org/conference-proceedings-of-spie on 26 Apr 2023
Terms of Use: https://www.spiedigitallibrary.org/terms-of-use}




\section{Authors}

Numbers in the index correspond to the last two digits of the seven-digit citation identifier (CID) article numbering system used in Proceedings of SPIE. The first five digits reflect the volume number. Base 36 numbering is employed for the last two digits and indicates the order of articles within the volume. Numbers start with 00, 01, 02, 03, 04, 05, 06, 07, 08, 09, 0A, 0B...0Z, followed by 10-12, 20-2Z, etc.

Adam, Jose M., 16

Aksnes, Astrid, 11

Angelova, Todora, $\mathrm{OQ}$

Arkwright, J. W., OK

Ashikbayeva, Zhannat, OT

Ataman, Çağlar, 05

Ayupova, T., OU

Baker, Noah R., 1E

Banerjee, Partha P., $0 Z$

Barton, John, OS

Beisenova, Aidana, OT, 18

Bekmurzayeva, A., OU

Beltrán Bernal, Lina Marcela, $1 \mathrm{H}$

Belz, Mathias, $1 \mathrm{~K}$

Blanc, Wilfried, OT, OU, 18

Calderón, Pedro A., 16

Canbaz, Ferda, $1 \mathrm{H}$

Cano-Velázquez, Mildred S., OY

Carney, A. S., OK

Carver, Gary, OS

Catcheside, P., OK

Cattin, Philippe C., $1 \mathrm{H}$

Chanda, Sheetal, OS

Chen, Linxi, $\mathrm{OH}$

Chopra, Aditi, $1 \mathrm{~J}$

Chung, Phil-Sang, OL

Cook, P. R., OK

Deng, Cong, $\mathrm{OZ}$

Duan, Liangcheng, 17

Dukenbayev, R., OU

Dumitrascu, Carla, $0 Q$

Eastwood, P., OK

Ewing, Kenneth J., 09

Floris, Ignazio, 16

Friederich, Niklaus F., $1 \mathrm{H}$

Gilmore, Adam M., $\mathrm{OH}$

Grattan, K. T. V., 1K

Gupta, Manish, OS

Gupta, Pooja, 1B, 1J

Hansen, Karolyn, $\mathrm{OZ}$

Hasegawa, Nobory, 00

Haus, Joseph, $\mathrm{OZ}$

Hernández-Cordero, Juan, OY

Hjelme, Dag Roar, 11

Hsieh, Chun-Yen, 02

Hua, Liwei, IF

Hubbi, Basil, 13

Issatayeva, Aizhan, 0T, 18

Iwai, Katsumasa, 15
Jelbuldina, Madina, OT

Jernelv, Ine L., 11

Jung, ChangHyun, OL

Kikuchi, Shunsuke, 00

Kim, Hyunjoo, OL

Kim, Jaesun, OL

Kitamura, Toshiyuki, 00

Klein, Karl-Friedrich, 1K

Korganbayev, Sanzhar, OT, OU

Lee, HyeYeon, OL

Lee, Junho, OL

Lei, Jincheng, $1 \mathrm{~F}$

Liao, Chun-Da, 02

Liu, Xuan, 13

Liu, Yuwei, 13

Llorente, Roberto, $\mathrm{OQ}$

Locknar, Sarah, OS

López-Marín, Luz M., OY

Lu, Ying, 17

Madrigal, Javier, 16

Major, Kevin J., 09

Matsuura, Yuji, 15

Mercadé, Laura, $0 Q$

Mikami, Katsuhiro, 00

Miyagi, Mitsunobu, 15

Molardi, Carlo, OT, OU, 18

Mondal, Samir K., 1B, 1J

Morant, Maria, $\mathrm{OQ}$

Nagura, Takeo, 00

Nakashima, Daisuke, 00

Nelson, Leonard Y., $1 \mathrm{E}$

Nishikino, Masaharu, 00

Ouh, ChiHwan, OL

Pandey, Purnendu Shekhar, 06, 19

Park, GaYe, OL

Prakash, Om, 06

Raghuwanshi, Sanjeev Kumar, 06, 19, 1A

Rhee, Yun-Hee, OL

Sadhu, Ahana, IA

Saini, Pankaj Kumar, 06

Sales, Salvador, 16

Sanghera, Jasbinder S., 09

Seibel, Eric J., $1 \mathrm{E}$

Shadab, Azhar, 06, 19

Shaimerdenova, M., OU

Shi, Yi-Wei, 15

Singh, Yadvendra, 06, 19, 1A

Sinha, Uttam, $0 Z$

Sovetov, Sultan, OT 
Strøm, Karina, 11

Sun, Tong, IK

Sypabekova, M., OU

Takaku, Hiroyuki, 15

Tiwari, Umesh, 1 J

Tosi, Daniele, OT, OU, 18

Vairagi, Kaushal, 1B, $1 \mathrm{~J}$

Vilches, Sergio, 05

Wall, A. J., OK

Wang, Guangyao, 17

Wang, Wei-Chih, 02

Werner, Jan, $1 \mathrm{~K}$

Wu, Yongji, $1 \mathrm{~F}$

Xiao, Hai, IF

Yao, Jianquan, 17

Zam, Azhar, $1 \mathrm{H}$

Zappe, Hans, 05

Zhang, Qi, IF

Zhu, Xiao-Song, 15

Zhu, Xuran, $1 \mathrm{~F}$ 


\section{Conference Committee}

Symposium Chairs

Jennifer K. Barton, The University of Arizona (United States)

Wolfgang Drexler, Medizinische Universität Wien (Austria)

Program Track Chairs

Tuan Vo-Dinh, Fitzpatrick Institute for Photonics, Duke University (United States)

Anita Mahadevan-Jansen, Vanderbilt University (United States)

Conference Chair

Israel Gannot, Johns Hopkins University (United States) and Tel Aviv University (Israel)

Conference Program Committee

Olga Bibikova, art photonics GmbH (Germany)

James P. Clarkin, Polymicro Technologies, A Subsidiary of Molex Incorporated (United States)

Ilko llev, U.S. Food and Drug Administration (United States)

Jin U. Kang, Johns Hopkins University (United States)

Karl-Friedrich Klein, Technische Hochschule Mittelhessen (Germany)

Pierre Lucas, The University of Arizona (United States)

Yuji Matsuura, Tohoku University (Japan)

Katy Roodenko, MAX IR Laboratories, LLC (United States)

Angela B. Seddon, The University of Nottingham (United Kingdom)

\section{Session Chairs}

1 Fiber Optic Sensors: Development and Characterization I

Katy Roodenko, MAX IR Laboratories, LLC (United States)

2 Fiber Optic Tools for Medical Applications I

Katy Roodenko, MAX IR Laboratories, LLC (United States)

3 Environmental Sensing

Katy Roodenko, MAX IR Laboratories, LLC (United States)

$4 \quad$ Fiber Optic Tools for Medical Applications II

Pierre Lucas, The University of Arizona (United States) 
5 Fiber Optic Sensors: Development and Characterization II Yuji Matsuura, Tohoku University (Japan)

6 Fiber Optic Tools for Medical Applications III James P. Clarkin, Polymicro Technologies (United States)

$7 \quad$ Fiber Optic Tools for Medical Applications IV Viacheslav G. Artyushenko, art photonics GmbH (Germany)

8 Fiber Optic Sensors: Development and Characterization III Karl-Friedrich Klein, Technische Hochschule Mittelhessen (Germany) 